\title{
HIPERBILIRRUBINEMIA NEONATAL: REVISIÓN DE LA SITUACIÓN ACTUAL.
}

\section{NEONATAL HYPERBILIRUBINEMIA: REVIEW OF THE}

\section{CURRENT SITUATION.}

\section{Autores:}

María José Castaño Picó a

Miriam Sánchez Maciá b

a Servicio de Neonatología, hospital General Universitario de Elche, Departamento de Salud 20.

b Servicio de Cirugía General y Cirugía Vascular, hospital General Universitario de Elche, Departamento de Salud 20.

\section{RESUMEN}

Oвjetrvo: identificar la incidencia de hiperbilirrubinemia neonatal.

Méтodo: estudio bibliométrico descriptivo transversal, realizado mediante búsquedas bibliográficas en la Biblioteca Virtual en Salud, en la Web of Science y en Medline con las palabras clave "hiperbilirrubinemia neonatal"/"neonatal hyperbilirubinemia" "ictericia neonatal"/"neonatal jaundice" "epidemiología" /"epidemiology" "incidencia"/"incidence"; en el periodo comprendido entre 01-01-2007 hasta 31 31-12-2010. Se excluyeron los 
artículos sobre hiperbilirrubinemia neonatal prolongada, hiperbilirrubinemia neonatal extrema y exanguinotransfusión. No hubo límites en el idioma.

Resultado: En Estados Unidos, un $15.6 \%$ de los recién nacidos presentan ictericia neonatal, y en Nigeria fue el 6'7\%. En Europa, la hiperbilirrubinemia neonatal varía desde el $59 \%$ hallado en Suecia hasta el $28^{\prime} 5 \%$ en Roma, el $11 \%$ encontrado en Portugal, 0 el $6 \%$ de Grecia. La incidencia de hiperbilirrubinemia neonatal en Pakistán es de 39'7/1.000, mientras que en Dinamarca es de 1'4/100.000 nacimientos a término o prematuros tardíos. La incidencia en España se sitúa en 1'93/10.000 en 2007 y en 1'69/10.000 en 2009. La incidencia de hiperbilirrubinemia severa en el Reino Unido es de 7'1/100.000.

Conclusiones: Existe una elevada variabilidad debido al origen multicausal de la enfermedad. España presenta una baja incidencia de hiperbilirrubinemia neonatal, aunque la menor incidencia encontrada corresponde a Dinamarca.

Palabras clave: Hiperbilirrubinemia neonatal, ictericia neonatal, epidemiología, incidencia.

\section{ABSTRACT}

Title: Neonatal hyperbilirubinemia: review of the current situation. Objective: to determine the incidence of neonatal hyperbilirubinemia.

Design: Bibliometric transverse descriptive study realized by literature searching in the Virtual Health Library, Web of Science and Medline with the keywords "hiperbilirrubinemia neonatal" / "neonatal hyperbilirubinemia" "ictericia neonatal"/"neonatal jaundice" "epidemiología "/"epidemiology" "incidencia"/" incidence" in the period understood between 01-01-2007 up to 3। 31-12-2010. We excluded articles on neonatal hyperbilirubinemia prolonged, extreme neonatal hyperbilirubinemia and exchange transfusion. There were no limits in the language.

Results: In the United States, $15.6 \%$ of newborns develop neonatal jaundice, and Nigeria is 6'7\%. In Europe, neonatal hyperbilirubinemia varies from $59 \%$ found in Sweden to $28.5 \%$ in Rome, $11 \%$ in Portugal, or $6 \%$ in Greece. The incidence of neonatal hyperbilirubinemia in Pakistan is $39.7 / 1.000$, while in Denmark is 1.4 per 100.000 births at term or near preterm. The incidence in Spain stands at $1^{\prime} 93 / 10.000$ in 2007 and $1^{\prime} 69 / 10.000$ in 2009. The incidence of severe hyperbilirubinemia in the United Kingdom is 7'1 / 100.000.

Conclusions: A high variability due to multi-causal origin of the disease. Spain has a low incidence of neonatal hyperbilirubinemia, although the lowest incidence was found within Denmark.

KeY words: Neonatal hyperbilirubinemia, neonatal jaundice, epidemiology, incidence 


\section{INTRODUCCIÓN}

La ictericia neonatal se caracteriza por una elevación de los niveles de bilirrubina sérica en los recién nacidos ${ }^{1}$, produciéndose una acumulación del pigmento amarillo-naranja de la bilirrubina en la piel, las escleróticas, y otros tejidos, sin que ello implique ninguna causalidad en particular ${ }^{2}$.

La hiperbilirrubinemia neonatal se debe principalmente a la acumulación de bilirrubina, como resultado de alteraciones metabólicas causadas por diversos factores ${ }^{3}$. Los recién nacidos, especialmente los pretérminos, tienen una conjugación y excreción de bilirrubina a través del hígado inmaduras ${ }^{2}$. Normalmente, los niveles de bilirrubina sérica aumentan entre el tercer y quinto día de vida, y luego comenzarán a disminuir ${ }^{3}$.

La ictericia neonatal es frecuente a nivel mundial ${ }^{4,5}$, y varía ampliamente entre diversas instituciones debido a diferencias raciales, prácticas de amamantamiento, condiciones hemolíticas ${ }^{4}$ o condiciones genéticas ${ }^{5,6}$. Los factores de riesgo en la hiperbilirrubinemia neonatal son la lactancia materna, la prematuridad, la incompatibilidad sanguínea ( $A B O$ o Rh), la infección, el cefalohematoma, la asfixia, el déficit de glucosa-6-fosfato deshidrogenasa, y las variantes genéticas que afectan a la actividad glucoronil-transferasa?.

En los estados patológicos, la bilirrubina sérica, especialmente la bilirrubina indirecta, puede alcanzar un altísimo nivel que lleva a la neurotoxicidad, provocando kernicterus, y finalmente daños neurológicos permanentes o incluso la muerte ${ }^{4}$.

Las medidas terapéuticas más habituales para el tratamiento de la hiperbilirrubinemia son la fototerapia y la exanguinotransfusión, puesto que ambos facilitan la eliminación de la bilirrubina producida por el organismo ${ }^{2,5}$.

El objetivo del artículo es conocer la incidencia de la hiperbilirrubinemia neonatal a nivel internacional mediante una búsqueda bibliográfica. 


\section{MATERIAL Y MÉTODOS}

Estudio bibliométrico, descriptivo transversal realizado mediante búsquedas bibliográficas en la Biblioteca Virtual en Salud, en la Web of Science y en Medline con las palabras clave "hiperbilirrubinemia neonatal"/"neonatal hyperbilirubinemia" "ictericia neonatal"/"neonatal jaundice" "epidemiología" /"epidemiology" "incidencia"/"incidence"; se seleccionó y consultó el material bibliográfico desde el 01-01-2007 hasta el 31-12-2010. Se excluyeron los artículos sobre hiperbilirrubinemia neonatal prolongada, hiperbilirrubinemia neonatal extrema y exanguinotransfusión. No hubo límites en el idioma. Los artículos seleccionados se clasificaron por relevancia, actualidad y localización.

\section{RESULTADOS}

El $60 \%$ de los recién nacidos a término y el $80 \%$ recién nacidos pretérmino presentan hiperbilirrubinemia durante la primera semana de vida $3,5,8$.

En Estados Unidos, aproximadamente el $60 \%$ de los recién nacidos (2'4 millones) desarrollan ictericia neonatal cada $a \tilde{n} 0^{9}$, habiéndose documentado ictericia neonatal en un $15.6 \%$ de los recién nacidos ${ }^{10}$.

En un reciente estudio italiano, realizado en Roma, el $28^{\prime} 5 \%$ de los prematuros tardíos (34-36+6 semanas de edad gestacional) presentaron ictericia que requirió tratamiento con fototerapia. Dicha ictericia apareció principalmente entre el $2^{\circ}$ y el $4^{\circ}$ día de vida y se resolvió sólo con fototerapia $^{11}$.

La incidencia de hiperbilirrubinemia severa en el Reino Unido es de 7'1 casos por cada 100.000 nacidos vivos, con una incidencia de encefalopatía bilirrubínica fue de 0 '9 casos por cada 100.000 habitantes $^{12}$.

En un estudio sueco, el $59 \%$ de la población estudiada presentaba hiperbilirrubinemia neonatal ${ }^{13}$. 
En Dinamarca se ha estimado una incidencia de 1'4 casos por cada 100.000 nacimientos a término o prematuros tardíos ${ }^{12}$.

En Pakistán, se encontró una incidencia de hiperbilirrubinemia neonatal de $39^{\prime} 7$ casos por cada 1.000 habitantes $^{14}$.

En un estudio realizado en Lagos (Nigeria), un 6'7\% presentó ictericia neonatal, de los cuales un $5^{\prime} 5 \%$ recibió fototerapia y un $1^{\prime} 9 \%$ exanguinotransfusión ${ }^{15}$.

En Canadá se estima que aproximadamente 5.000 recién nacidos a término desarrollan hiperbilirrubinemia severa cada año; en China se estima sobre 150.000 recién nacidos a término anuales ${ }^{16}$.

En un estudio realizado en Oporto (Portugal), el $11 \%$ presentaba hiperbilirrubinemia significativa ${ }^{17}$.

Un estudio realizado en Grecia halló una hiperbilirrubinemia neonatal significativa en el $6 \%$ de los recién nacidos a término y prematuros tardíos ${ }^{18}$.

En España, según datos proporcionados por el Ministerio de Sanidad y Política Social ${ }^{19}$, la incidencia de ictericia neonatal durante el año 2007 fue de 1'93 casos por cada 10.000 habitantes con una estancia media de 3'01 días, la incidencia en 2008 fue de 2'05 casos por cada 10.000 habitantes con una estancia media de 2'85 días; y en el año 2009 la incidencia fue de 1'69 casos por cada 10.000 habitantes con una estancia media de 2'78 días; la incidencia total en España durante el periodo 2007-2009 fue de 1'89 casos por cada 10.000 habitantes con una estancia media de 2'88 días. Dicha fuente también proporciona datos por comunidades autónomas, y en la Comunidad Valenciana, durante el año 2007, la incidencia de ictericia neonatal fue de 1'94 casos por cada 10.000 habitantes con una estancia media de 2'96 días; mientras que en el año 2008 la incidencia de ictericia neonatal fue de 2'10 casos por cada 10.000 habitantes y una estancia media de 2'84 días; y en el año 2009 la incidencia fue de $1^{\prime} 56$ casos por cada 10.000 habitantes y una estancia media de 2'61 días, mientras la incidencia en la Comunidad Valenciana durante el periodo 2007-2009 fue de 1'87 casos por cada 10.000 habitantes y una 
estancia media de 2'81 días.

Si clasificamos estos datos según sexo, la incidencia de ictericia neonatal en la Comunidad Valencia durante:

- el 2007 fue de 2'30 casos por cada 10.000 habitantes y una estancia media de 2'72 días en el caso de los varones, y en el caso de las mujeres la incidencia fue de 1'59 casos por cada 10.000 habitantes con una estancia media de 3'30 días

- el año 2008, la incidencia de ictericia neonatal en varones fue de 2'30 casos por cada 10.000 habitantes y una estancia media de 2'84 días, en cambio la incidencia en las mujeres fue de 1'90 casos por cada 10.000 habitantes con una estancia media de 2'83 días

- el año 2009 la incidencia fue de 1'79 casos por cada 10.000 habitantes y una estancia media de 2'46 días en el caso de los varones, mientras que en el caso de mujeres la incidencia fue de 1'33 casos por cada 10.000 habitantes con una estancia media de 2'80 días

La incidencia a nivel nacional según sexos se muestra en la tabla 1 (ver anexo).

La evolución de la incidencia de la hiperbilirrubinemia neonatal (casos/10.000 habitantes) en las distintas Comunidades Autónomas se presenta en la tabla 2 (ver anexo).

En un estudio realizado en Oviedo (Asturias) se encontró una incidencia de hiperbilirrubinemia neonatal de 5 casos por cada 1.000 habitantes $^{20}$.

\section{DISCUSIÓN}

La hiperbilirrubinemia severa tratada con exanguinotransfusión se ha convertido en una situación relativamente rara en la actualidad, por lo que se decidió excluirlo de la búsqueda; no obstante, la hiperbilirrubinemia moderada se observa en aproximadamente el $60 \%$ de los neonatos a término y en el $80 \%$ de los neonatos pretérmino durante la primera semana de vida debido a 
una menor vida media de las células rojas de la sangre y a la inmadurez de la función hepática 20 .

La hiperbilirrubinemia neonatal se puede agravar por la deshidratación debido a la falta de leche pero también pueden ocurrir en presencia de lactancia exitosa. Los recién nacidos prematuros tardíos nacen rondando la frontera de la prematuridad, y pueden ser vulnerables debido a la dificultad relativa en el establecimiento de la lactancia y la relativa inmadurez de la captación hepática y la conjugación de bilirrubina ${ }^{12}$.Se asocia tanto con hiperbilirrubinemia severa como con encefalopatía por bilirrubina el sexo masculino, pertenecer a minorías étnicas, la lactancia materna y la comorbilidad, incluyendo la deshidratación, los hematomas, las enfermedades hemolíticas, y la infección ${ }^{12}$. Según un reciente estudio, el ser madre obesa es un factor de riesgo para la hiperbilirrubinemia neonatal ${ }^{21}$.

Los niveles de bilirrubina aumentan entre el tercer y quinto día de vida ${ }^{18}$; no obstante, la práctica clínica en la vigilancia y el tratamiento de la ictericia neonatal varía ampliamente, probablemente porque muchos aspectos de la gestión no son compatibles con una firme evidencia ${ }^{12}$.

El hecho de nacer mediante cesárea y establecer una lactancia artificial no tienen efecto en la incidencia de la hiperbilirrubinemia significativa ${ }^{18}$.

Una limitación del presente estudio puede ser las diferentes definiciones $^{22}$ y clasificaciones de hiperbilirrubinemia que realizan los distintos autores.

Otra limitación del estudio es la obtención de datos específicos para un determinado nivel de hiperbilirrubinemia, por lo que las incidencias señaladas no incluirían todos los subtipos de hiperbilirrubinemia. Por otro lado, lo ideal sería que los datos proporcionados en los artículos fuesen todos del mismo año.

Un reciente artículo americano halló en los recién nacidos afroamericanos una baja incidencia de hiperbilirrubinemia neonatal, pero una elevada incidencia de kernicterus de más del $25 \%{ }^{23}$, por lo que la gestión del riesgo en 
la enfermedad y un adecuado tratamiento es vital para evitar en la medida de lo posible unas terribles consecuencias.

Durante los años 2007-2009, la Comunidad Valenciana presenta una incidencia de hiperbilirrubinemia neonatal de 1'87 casos por cada 10.000 habitantes, cifra similar a la incidencia global de España de 1'89 casos por cada 10.000 habitantes en el mismo periodo, y en ambos casos es mayor en el sexo masculino. Si comparamos éstas dos últimas cifras de incidencias con la encontrada en un estudio en Oviedo de 5 por cada 1.000 habitantes, la incidencia de la hiperbilirrubinemia neonatal en Oviedo es mucho más elevada; de todos los países, la menor incidencia se ha establecido en Dinamarca. La incidencia hallada más elevada corresponde a Pakistán, mientras que los datos de Portugal, Grecia, Suecia, Nigeria, Roma y Estados Unidos se establece en proporciones; en China y Canadá se habla de datos globales; y en el Reino Unido la incidencia es específica de la hiperbilirrubinemia severa.

\section{CONCLUSIONES}

Existe una elevada variabilidad en la incidencia de la hiperbilirrubinemia neonatal debido al origen multicausal de la enfermedad.

En la actualidad España, que se caracteriza por su gran accesibilidad a los servicios sanitarios, presenta una baja incidencia de hiperbilirrubinemia neonatal, aunque la menor incidencia encontrada corresponde a Dinamarca.

Para optimizar el manejo de la hiperbilirrubinemia neonatal, es necesaria una gestión del riesgo como herramienta para seleccionar, planificar y evaluar las estrategias adecuadas para el tratamiento de la enfermedad. 


\section{ANEXOS}

\begin{tabular}{|c|c|c|c|c|c|}
\hline & Tabla 1 & & & $\begin{array}{l}\text { Estancia } \quad \text { Media } \\
\text { en días }\end{array}$ & $\begin{array}{l}\text { Casos por } 10.000 \\
\text { habitantes. }\end{array}$ \\
\hline \multirow[t]{3}{*}{2007} & \multirow{3}{*}{$\begin{array}{l}\text { 774-OTRAS } \\
\text { PERINATAL }\end{array}$} & \multirow[t]{3}{*}{ ICTERICIAS } & Varón & 3,00 & 2,23 \\
\hline & & & Mujer & 3,03 & 1,63 \\
\hline & & & Otros & 3 & \\
\hline \multirow[t]{3}{*}{2008} & \multirow{3}{*}{$\begin{array}{l}\text { 774-OTRAS } \\
\text { PERINATAL }\end{array}$} & \multirow[t]{3}{*}{ ICTERICIAS } & Varón & 2,87 & 2,33 \\
\hline & & & Mujer & 2,82 & 1,78 \\
\hline & & & Otros & 4 & \\
\hline \multirow[t]{3}{*}{2009} & \multirow{3}{*}{$\begin{array}{l}\text { 774-OTRAS } \\
\text { PERINATAL }\end{array}$} & \multirow[t]{3}{*}{ ICTERICIAS } & Varón & 2,82 & 1,96 \\
\hline & & & Mujer & 2,73 & 1,42 \\
\hline & & & Otros & 2,71 & \\
\hline
\end{tabular}

Fuente: Ministerio de Sanidad, Politica Social e Igualdad. Instituto de Información Sanitaria. Registro de altas - CMBD.

\section{Tabla 2}

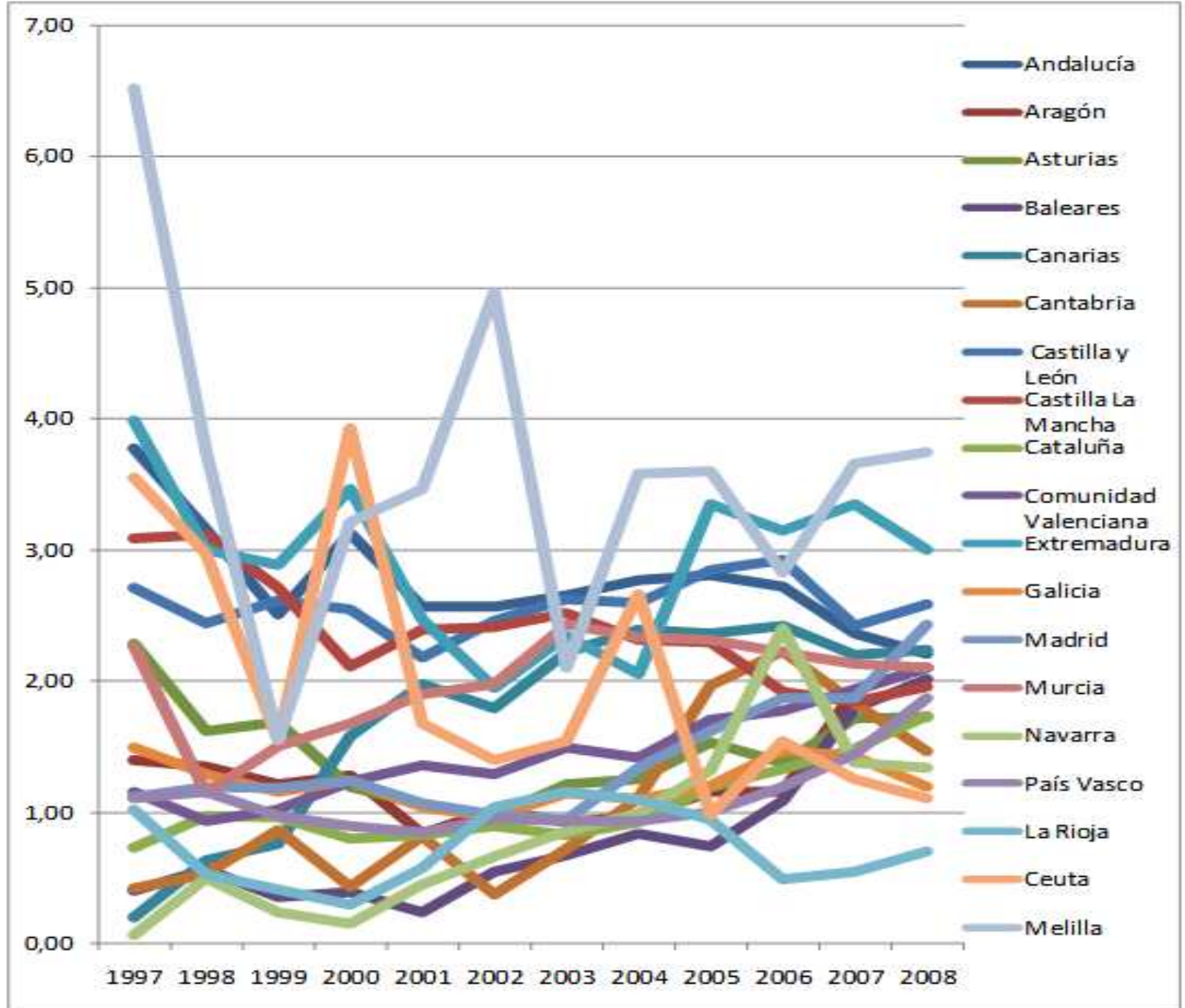

Fuente: Ministerio de Sanidad, Política Social e Igualdad. Instituto de Información Sanitaria. Registro de altas - CMBD. 


\section{REFERENCIAS BIBLIOGRÁFICAS}

1. Fernández Rodríguez M, Martín Muñoz P. La valoración clínica de la ictericia no es buen método para el cribado de hiperbilirrubinemianeonatal. EvidPediar. 2008; 4: 77.

2. Cohen RS, Ronald JW, Stevenson DK. Understanding Neonatal Jaundice: A Perspective on Causation. PediatrNeonatol. 2010; 51(3):143-48.

3. Wu J, Su W, Jin Y, Shi Y, Li C, Zhong W et al. Targeted suppression of heme oxygenase-1 by small interference RNAs inhibits the production of bilirubin in neonatal rat with hyperbilirubinemia. BMC Mol Biol. 2009; 10: 77.

4. Puebla Molina SF, Aparicio Sánchez JL. En el tratamiento de la ictericia neonatal, el uso de cortinas blancas reflectantes de bajo coste alrededor de la zona de fototerapia incrementa significativamente la eficacia de ésta. EvidPediatr. 2007; 3:18.

5. Maisels MJ, McDonagh AF. Phototherapy for Neonatal Jaundice. N Engl J Med. 2008; 358:920-8.

6. Watchko JF, Lin ZL. Exploring the genetic architecture of neonatal hyperbilirubinemia.Semin Fetal Neonatal Med. 2010; 15 (3): 169-75.

7. Checa DA. Manual DAE de enfermería; EIR; Oposiciones. $1^{\text {a }}$ ed. Madrid: Difusión Avances de Enfermería; 2009.

8. Rennie J, Burman-Roy S, Murphy S. Neonatal jaundice: summary of NICE guidance. BMJ. 2010; 340:c2409.

9. Huang MS, Lin $\mathrm{MC}$, Chen $\mathrm{HH}$, Chien $\mathrm{KL}$, Chen $\mathrm{CH}$. Risk Factor Analysis for Late-onset Neonatal Hyperbilirubinemia in Taiwanese Infants. PediatrNeonatol. 2009; 50(6):261-65.

10. Burke BL, Robbins JM, Mac Bird T, Hobbs CA, Nesmith C, Tilford JM. Trends in Hospitalizations for Neonatal Jaundice and Kernicterus in the United States, 1988-2005. Pediatrics. 2009; 123(2):524-32.

11. Picone S,Paolillo P. Neonatal outcomes in a population of late-preterm infants. J Matern Fetal Neonatal Med. 2010; 23(S3):116-20.

12. Manning $D$, Todd $P$, Maxwell M, Plait MJ. Prospective surveillance study of severe hyperbilirubinaemia in the newborn in the UK and Ireland.Arch Dis Child Fetal Neonatal Ed.2007; 92(5):342-46.

13. Altman M, Vanpée $M$, Cnattingius $S$, Norman $M$. Neonatal morbidity in moderately preterm infants: a Swedish national population-based study.J Pediatr. 2011; 158(2):239-44.

14. Tikmani SS, Warraich HJ, Abbasi F, Rizvi A, Darmstadt GL, Zaidi AKM. Incidence of neonatal hyperbilirubinemia: a population-based prospective study in Pakistan.Trop Med Int Health.2010; 15(5): 502-07.

15. Olusanya BO, Akande AA, Emokpae $A$, Olowe SA. Infants with severe neonatal jaundice in Lagos, Nigeria: incidence, correlates and hearing screening outcomes. Trop Med Int Health. 2009; 14(3): 301-10.

16. Sankaran K. Hyperbilirubinemia in newborns with gestation $\geq 35$ weeks. Zhongguo Dang Dai ErKeZaZhi. 2010; 12(10):761-6.

17. Gonçalves A, Costa S, Lopes A, Rocha G, Guedes MB, Centeno MJ et al. Prospective validation of a novel strategy for assessing risk of significant 
hyperbilirubinemia. Pediatrics. 2011; 127(1):e126-31.

18. Varvarigou A, Fouzas S, Skylogianni E, Mantagou L, Bougioukou D, Mantagos S. Transcutaneous bilirubin nomogram for prediction of significant neonatal hyperbilirubinemia. Pediatrics. 2009; 124; 1052-59.

19. Consulta interactiva SNS [Internet]. Madrid: Ministerio de Sanidad, Política Social e Igualdad; [acceso 8 febrero 2011]. Instituto de Información Sanitaria. Registro de altas - CMBD. Disponible en: http://pestadistico.msc.es

20. Núñez-Batalla F, Carro-Fernández $P$, Antuña-León ME, González-Trelles T. Incidencia de hipoacusia secundaria a hiperbilirrubinemia en un programa de cribado auditivo neonatal universal basado en otoemisiones acústicas y potenciales evocados auditivos.Acta Otorrinolaringol Esp. 2008;59(3):108-13.

21. Briese V, Voigt M, Hermanussen M, Wittwer-Backofen U.Morbid obesity: Pregnancy risks, birth risks and status of the newborn. Homo. 2010; 61(1):64-72.

22. Trikalinos TA, Chung M, Lau J, Ip S. Systematic review of screening for bilirubin encephalopathy in neonates. Pediatrics. 2009; 124: 1162-71.

23. Watchko JF. Hyperbilirubinemia in African American neonates: clinical issues and current challenges. Semin Fetal Neonatal Med.2010; 15(3): 176-82. 